\title{
Final Report 2007: DOE-FG02-87ER60561
}

Efforts of this research project resulted in diverse advances in (a) synthesis of new radiotracers for neurochemical studies, (b) evaluation of microPET imaging to uncover unusual transient effects of drugs, (c) development of new radiotracers to investigate biochemistry of cancers, (d) human studies using single-session dual-radiotracer imaging paradigms, and (e) continued development of improved image processing and analysis methods.

Evaluation of 1- $\left[{ }^{18} \mathbf{F}\right]$ fluoroethyl analogs of cholinesterase substrates. We prepared a series of $\left[{ }^{18} \mathrm{~F}\right]$ fluoroethyl compounds analogous to $N-\left[{ }^{11} \mathrm{C}\right]$ methyl-4-piperidinyl acetate $\left(\left[{ }^{11} \mathrm{C}\right] \mathrm{PMP}\right)$ and determined the in vitro cleavage rates in purified $\mathrm{AChE}$ and BuChE, rate of metabolism in mouse blood, ex vivo biodistribution in mouse brain and in vivo the behavior in rats measured by microPET imaging. Four candidate radiotracers, $(S)$ - $N$-fluoroethyl-2-piperidinemethyl acetate $(\mathbf{1}),(R)$ - $N$-fluoroethyl-3-pyrrolidinyl acetate (2), $N$-fluoroethyl-4-piperidinyl acetate $(3)$ and $(R)-N$-fluoroethyl-3-piperidinyl acetate (4), were evaluated. Compound 1 showed rapid metabolism in mouse blood, relatively fast regional brain pharmacokinetics and poor discrimination between brain regions with different $\mathrm{AChE}$ concentration (striatum versus cortex). Compound $\mathbf{4}$ showed relatively slower blood metabolism and slower pharmacokinetics than compound $\mathbf{1}$, but again poor discrimination between brain regions. Compound $\mathbf{3}$ had the slowest blood metabolism and pharmacokinetics too slow to be practical for imaging. Compound $\mathbf{2}$ showed highly encouraging characteristics with an in vivo metabolism rate slower than $\left[{ }^{11} \mathrm{C}\right] \mathrm{PMP}$, similar primate brain uptake and pharmacokinetics to $\left[{ }^{11} \mathrm{C}\right] \mathrm{PMP}$, and good discrimination between brain regions.

Development of HPLC methods for measuring $\log \boldsymbol{k}_{\mathrm{w}}$ and analysis of model compounds. We have developed a standard HPLC method for predicting blood-brain barrier permeation of tracer candidates, based on tracer lipophilicity under physiological conditions. Many radiotracer molecules contain ionizable functional groups (such as amines), and the HPLC system used must mimic physiological $\mathrm{pH}$ and ionic strength to be an accurate index of BBB permeation. After testing a variety reversed-phase and ionpairing HPLC columns, and numerous combinations of solvents and buffer salts, we settled on a standard Ultremex $5 \mu \mathrm{m} \mathrm{C18} \mathrm{column}(4.6 \times 250 \mathrm{~mm})$ and a mixture of acetonitrile and $100 \mathrm{mM} 3$-( $N$-morpholino)propanesulfonic acid (MOPS) buffer at $\mathrm{pH}$ 7.4. This system allows analysis of a wide range of compounds at UV wavelengths as low as $220 \mathrm{~nm}$, and was then used to measure the capacity factor of several test compounds at three different solvent mixtures (i.e. \% acetonitrile). The mean capacity factor $(k)$ at each solvent strength $(\mathrm{n}=3-5)$ was determined, where $k=\left(\mathrm{t}_{\mathrm{R}}-\mathrm{t}_{0}\right) / \mathrm{t}_{0}$, and $\mathrm{t}_{0}$ is the column dead time based on the elution time of methanol as an unretained species. The $\log k$ values in each solvent mixture were then plotted versus \% acetonitrile and extrapolated to provide $\log k_{\mathrm{W}}$, the capacity factor at $0 \%$ acetonitrile. The $\log k_{\mathrm{W}}$ values of dihydrotetrabenazine (DTBZ), flumazenil (FMZ), phenethylamine, tyramine and methyl 1-methylisonipecotate have been measured. So far, there is no correlation with reported (or calculated) $\log \mathrm{P}$ values, however several more compounds will need to be analyzed before valid correlations with lipophilicity $(\log \mathrm{P})$ or BBB permeation (as measured by initial brain uptake of radiotracers in rodents) can be determined. 
MicroPET: Effects of anesthesia on in vivo neurochemical measures. One goal of this project is to measure the changes in trafficking of the dopamine transporter via manipulations of the cholinergic system. The use of microPET as the analytical method to measure cholinergic drug-induced changes requires (1) demonstration that anesthesia, currently required for routine small animal microPET studies, does not interfere with the drug-induced biochemical changes, and (2) that microPET is capable of measuring small changes in radiotracer binding. We have thus completed a study comparing the drug induced changes in the binding of a muscarinic cholinergic antagonist radioligand, $\mathrm{N}-\left[{ }^{11} \mathrm{C}\right]$ methylpyrrolidinylbenzilate (NMPYB), in awake and anesthetized animals using both microPET and classical tissue dissection techniques. All ex vivo dissection studies were done using the previously established bolus+infusion method of radiotracer administration, which allows determination of equilibrium distribution volume ratios (DVR) in regions of interest such as the striatum, cortex and hippocampus (cerebellum used as reference region for nonspecific distribution). In both the awake and anesthetized state, we found that administration of the acetylcholinesterase inhibitor phenserine induced a 9-11\% decrease in the in vivo specific binding of $\left[{ }^{11} \mathrm{C}\right] \mathrm{NMPYB}$ in the striatum (control DVR $=6.84+/-0.32$; phenserine-treated $6.29+/$ $0.41, \mathrm{P}<0.05$ ) and cortex (control DVR $=5.36+/-0.15$; phenserine treated $4.79+/-0.36$, $\mathrm{P}<0.05)$. These small changes were evident with a relatively small number of animals per experimental group $(\mathrm{N}=4$ or 5). It was thus clear that anesthesia does not interfere with the actions of an acetylcholinesterase inhibitor to raise brain acetylcholine levels in the rat.

When this experiment was performed using the microPET as the instrument of analysis, it was not possible to measure the expected decrease in binding of $\left[{ }^{11} \mathrm{C}\right]$ NMPYB. Studies were done using bolus injection of radiotracer, imaging of radiotracer distribution for one hour, and pharmacokinetic modeling of the regional tissue time-activity curves using a reference region Logan plot using the cerebellum as the reference tissue. There were no significant differences seen between control (striatal DVR $=2.80+/-0.37, \mathrm{~N}=5$ ) and phenserine-treated animals (striatal DVR $=3.10+/-$ $0.42, \mathrm{~N}=5$ ). As we had shown that the anesthesia needed for the microPET studies should not have interfered, our conclusion is that the microPET method was not sensitive enough to measure small (10\%) changes in radiotracer binding in a reasonably small group of animals. It remains undetermined if small changes in radiotracer binding might be measured using a large number of animals.

We then examined the in vivo binding of $N-\left[{ }^{18} \mathrm{~F}\right]$ fluoroethyl-piperidinyl benzilate $\left(\left[{ }^{18} \mathrm{~F}\right] \mathrm{FEPB}\right)$ to the muscarinic cholinergic receptor was measured in awake and anesthetized rats. Studies were done using an equilibrium infusion technique to provide estimates of specific binding as distribution volume ratios. Anesthesia with either isoflurane or sodium pentobarbital produced a significant (65-90\%) increase of radiotracer binding in receptor-rich brain regions (striatum, cortex, hippocampus) relative to awake controls. Pretreatment of anesthetized animals with the acetylcholinesterase inhibitor phenserine produced no further increases in radioligand binding, in contrast to the large (>70\%) increases previously observed in awake animals following drug treatment. These studies demonstrate that anesthesia can produce significant changes in baseline biochemical measures that can obscure even very large effects of pharmacological challenges. 
MicroPET: Imaging partial lesions of the striatal dopaminergic system. The ability of the microPET to differentially image and quantify dopaminergic terminal loss in partially-lesioned rat striata was evaluated 8 animals. All received partial 6hydroxydopamine lesions of the right medial forebrain bundle (MFB), producing unilateral lesions in the contralateral striatum. Each animal was then imaged using $\left[{ }^{11} \mathrm{C}\right]$ dihydrotetrabenazine (DTBZ) and microPET. Using bolus injection of the $\left[{ }^{11} \mathrm{C}\right] \mathrm{DTBZ}$, one hour of imaging, and Logan plot analyses, distribution volume ratios for right and left striata were obtained. There was a consistent ( $8 / 8$ animals) reduction in striatal DVR on the lesioned side of all animals: control striatal DVR $=6.18+/-0.79$; lesioned striatal $\mathrm{DVR}=5.54+/-1.05, \mathrm{P}<0.01$ paired $\mathrm{t}$-test). The average loss of binding of about $10 \%$ (lesioned/control $=0.89+/-0.08$; range 0.72 to 0.95 ).

Scopolamine: Differential effects on monoamine transporters. We have studied the effects of scopolamine, a muscarinic cholinergic antagonist, on the in vivo binding of radiotracers for transporters in monoaminergic neurons. We had previously found that scopolamine increased $\left[{ }^{3} \mathrm{H}\right]$ methylphenidate binding to the DAT (controls, striatal DVR $=2.11+/-0.15$; scopolamine-treated, DVR $=2.44+/-0.25, \mathrm{p}<0.05)$, and hypothesized that this was due to a indirect effect of scopolamine on intracellular levels of protein kinase $\mathrm{C}$. We have now shown that scopolamine does not have any effect on in vivo binding of $\left[{ }^{11} \mathrm{C}\right] \mathrm{DTBZ}$ to the VMAT2 (controls, striatal DVR $=3.29+/-0.23$; scopolamine-treated, DVR $=3.39+/-0.48, \mathrm{p}>0.50)$. This is consistent with our hypothesis that VMAT2 is not a regulated transporter, and supports that the changes seen in the methylphenidate study were not due to such as altered blood flow.

Dual Radiotracer Studies: Enhancement of sensitivity to biochemical changes. Using a data set for simultaneous $\left[{ }^{11} \mathrm{C}\right] \mathrm{DTBZ}$ (VMAT2 radioligand) and $\left[{ }^{3} \mathrm{H}\right]$ methylphenidate (DAT radioligand) equilibrium infusions, the ratio of striatal DVR ratios for $\left[{ }^{3} \mathrm{H}\right]$ methylphen/ $\left[{ }^{11} \mathrm{C}\right] \mathrm{DTBZ}$ was calculated for control animals as well as animals treated with a dopamine antagonist and a dopamine agonist. In control animals, the ratio in control animals was $0.54+/-0.02$. In haloperidol treated animals, the ratio was $0.52+/-0.04$. The apomorphine-treated animals showed a ratio of $0.74+/-0.21$, a significant change relative to controls $(\mathrm{P}=0.04)$ and haldol-treated animals $(\mathrm{P}=0.03)$. The observed increase in the methylphen/DTBZ ratio could correspond to an increase in DAT availability, and this would fit our proposed model of trafficking of the DAT between cytosolic and external membrane locations.

Drug sensitivity of VMAT2 and DAT radioligand binding: dopamine

depletion. We have completed a study of the effects of dopamine depletion on differential drug sensitivity of DAT and VMAT2 radioligands in normal rat brain. The sensitivity of the in vivo binding of $\left[{ }^{11} \mathrm{C}\right]$ dihydrotetrabenazine and $\left[{ }^{11} \mathrm{C}\right]$ methylphenidate to their respective targets, the vesicular monoamine transporter (VMAT2) and the neuronal membrane dopamine transporter (DAT), after alterations of endogenous levels of dopamine were examined in the rat brain. Repeated dosing with $\alpha$-methyl-p-tyrosine (AMPT) at doses sufficient to nearly completely deplete dopamine levels resulted in increased $(+26 \%)$ in vivo $\left[{ }^{11} \mathrm{C}\right]$ dihydrotetrabenazine binding in the striatum. The increase in binding could be reversed by treatment with L-DOPA/benserazide to restore dopamine levels. There were no changes in total numbers of vesicular monoamine transporter binding sites as measured using in vitro autoradiography. No changes were observed for in vivo $\left[{ }^{11} \mathrm{C}\right]$ methylphenidate binding to the dopamine transporter in the striatum 
following $\alpha$-methyl-p-tyrosine pretreatment. These results indicate that large changes of dopamine concentrations in the rat brain can produce small but significant changes in binding of radioligands to the vesicular monoamine transporter.

MicroPET imaging of dynamic effects of AMPT on $\left[{ }^{11} \mathrm{C}\right] \mathrm{DTBZ}$ binding. The ability of microPET imaging to measure the above changes in $\left[{ }^{11} \mathrm{C}\right] \mathrm{DTBZ}$ bidning after AMPT treatment was evaluated. The equilibrium infusion protocol for radiotracer administration was first validated in a set of control animals $(\mathrm{N}=14)$ to provide a composite tissue time-radioactivity curve with stable striatum-to-cerebellum ratios for the period of 45-90 minutes. At equilibrium this ratio (2.84) represents the distribution volume for the striatum, a measure of the specific binding. Surprisingly, injection of AMPT at 40 minutes after beginning the radioligand infusion into a second set of animals $(\mathrm{N}=14)$ produced an unusual initial decrease in the str/cbl ratios lasting for 15 minutes, after which the str/cbl ratios rose and at the end of the study were equivalent (2.82) to the control animals. This effect of AMPT was significantly different than had been observed in earlier dissection experiments.

The transient decrease of the tissue time-radioactivity curves following AMPT injection, seen for every animal, remains puzzling and unexplained. In almost all cases, animals were done in pairs (one control, one AMPT-treated) to remove confounding effects of different radiochemical preparations, and scanners (microPET R4 and P4) randomized to remove that confound. The transient changes were not seen with injection of other dopaminergic drugs, such as haloperidol and clorgylline. The transient inflection in the curve was not seen upon AMPT administration during an infusion of

$\left[{ }^{11} \mathrm{C}\right]$ methylphenidate, a dopamine transporter (DAT) radioligand. This unusual behavior of the [C-11]DTBZ upon acute severe depletion of dopamine levels continues to be under study.

PepT1 transporter activity as a target for tumor imaging in pancreatic cancer with
[C-11]Glycylsarcosine ([C-11]Gly-Sar).Pancreatic cancer is one of the most aggressive human tumors, with a 5 year survival rate of only 3-5\% and a median survival of less than 6 months. Early detection and accurate staging are critical for effective management and optimal treatment planning in this disease. However, none of the currently available PET radiopharmaceuticals are useful for imaging pancreatic cancer. The proton-coupled di/tri-peptide transporter, PEPT1 is highly expressed in a number of immortalized human tumor cell lines, including several pancreatic adenocarcinoma lines. Recent work in this project has focused on determining the utility of measuring expression of the PEPT1 proton-coupled di/tri-peptide transporter in the diagnosis, staging and therapy planning in adenocarcinoma of the pancreas. Preliminary tumor imaging studies were performed with the radiolabeled PEPT1 substrate, [C-11] Gly-Sar in four pancreatic (AsPC-1, Capan-2, MPanc-96 and PANC-1) and one prostate (PC-3) adenocarcinoma cell lines expressing a range of PEPT1 activity. The initial uptake of [C-11]Gly-Sar in the various tumor types, measured by ex vivo dissection and counting methods, agreed with the rank order of PEPT1 expression measured in vitro by RT-PCR and Western blot analysis. By 60 minutes post-injection differences in tracer retention were statistically significant between the high (MPanc-96, AsPC-1, and Capan-2) and low (PANC-1 and PC-3) PEPT1 expression groups $(\mathrm{p}<0.001)$. However, all tumors retained radioactivity 1.5 (PC-3) to 3-fold (MPanc, AsPC-1) above blood levels at this later time point. Dual tracer ([C-11]Gly-Sar/[F-18]FDG) imaging experiments were also consistent with in vitro PEPT1 expression measurements. MPanc-96 showed highest retention of radioactivity, 
followed by AsPC-1 and Capan-2. PANC-1 and PC-3 tumors exhibited the lowest radioactivity. These differences between high (MPanc-96), intermediate (AsPC-1, Capan2) and low (PANC-1, PC-3) [C-11]Gly-Sar retention were statistically significant $(\mathrm{p}(\mathrm{High} / \mathrm{Mid})=0.08, \mathrm{p}(\mathrm{Mid} / \mathrm{Low})<0.005)$. The $[\mathrm{F}-18] \mathrm{FDG}$ retention in these tumor lines was uniformly higher than that of [C-11]Gly-Sar and exhibited an equally large variance between individual tumors. Differences in the [F-18]FDG retention between tumors were obvious in this series, however as expected these differences do not appear to correlate with PEPT1 activity. Taken together this set of in vitro, ex vivo and in vivo experiments provide strong evidence that [C-11]Gly-Sar uptake and retention in tumors is mediated by PEPT1 transport. Furthermore, it should be possible to measure moderate differences between PEPT1 activity levels in vivo with PET. This represents a novel new method for imaging and potential biochemical characterization of pancreatic tumors.

Dual Radiotracer Overlapping Studies: Human PET studies. The general aim of this project is to estimate multiple neuropharmacological parameters within a single noninvasive positron emission tomography (PET) procedure. The significance of this research centers on the concept that rather than examining particular aspects of the working brain in isolation, we would like to provide a broader more global view of cerebral function. We investigated an experimental protocol for dual-injection, singleacquisition PET scans that does not require arterial blood sampling. We have performed a variety of computer simulations to determine how best to estimate model parameters from a dual-injection study without the use of measured arterial input functions. The procedure has several steps including the use of various reference region-type calculations (Logan plots, reference tissue model), some with limited temporal ranges of data. The simulations performed so far have given us an idea of how much additional variability to expect in the estimates of the parameters for both tracers, relative to singletracer studies. We have also been working on ways to improve precision through various constraints or estimation parameters across multiple regions simultaneously.

A total of 76 subjects have received dual-tracer PET studies performed using three different combinations of radiotracers; i) [C-11]flumazenil (FMZ) with [C11]dihydrotetrabenazine (DTBZ); and ii) [C-11]FMZ with N-[ C-11]methylpiperidinyl propionate (PMP) and [C-11] raclopride (RAC) with [C-11] methylphenidate (MPH). The first and third combinations of radiotracers each involve one tracer with rapidly reversible kinetics (FMZ or RAC) and one with moderate kinetics (DTBZ or MPH), but still reversible kinetics. The second pair involves one tracer with rapidly reversible kinetics, but the second (PMP) with irreversible kinetics (i.e. $\mathrm{k} 4=0$ ). In the first two years of the grant, both a dual-tracer and a single-tracer PET study were been performed on each individual for comparison and validation of the dual-tracer results using the single-tracer study as a gold standard. Subsequently two dual-tracer studies have been performed on each subject, separated in time by one day up to one week. These studies involve one baseline dual-tracer scan and one scan acquired under a condition of partial blocking of one of the tracers (FMZ) with a non-radioactive antagonist (Romazicon). The purpose of these studies is to validate that the kinetic model parameters change significantly between scans only for the tracer whose uptake is blocked by the antagonist (FMZ), while the other tracer (either DTBZ or PMP) parameters are statistically unchanged. In the past year of the project 28 of these subjects have had two dual-tracer scans performed. Results show both good sensitivity to the antagonist-induced changes in binding in the one tracer and good specificity where the other tracer was unaffected. 
The key to good performance of a non-invasive dual-tracer PET protocol is the separation of the two tracer components of the total PET uptake curves. New methods to separate these curves are being explored in an attempt to reduce bias and improve precision in the kinetic parameter estimates.

New reconstruction algorithms for microPET. We developed imaging reconstruction algorithms for PET, with an emphasis on dynamic PET imaging. We have completed the development, analysis, and evaluation of a new algorithm for reconstructing PET images from PET scans that precorrected for random coincidences by real-time subtraction of delayed-window counts. This subtraction produces negative sinogram values that are incompatible with the usual Poisson statistical model used in PET image reconstruction. The conventional "solution" to this problem, used in some commercial PET systems, is to set the negatives values to zero and pretend that the data is Poisson. Raising negative values to zero can introduce a positive bias in activity quantification. We have developed new iterative reconstruction algorithms that account for the effect of subtracted delayed coincidences and allow for negative sinogram elements. Both theoretical analysis and computer simulations show that the new algorithms avoid the bias of the conventional method yet also have good noise properties. The computation time of the new method is equivalent to those of existing methods used clinically, so we have recommended the new methods for routine use for randomsprecorrected PET scans.

\section{Publications:}

Nabulsi NB, Smith DE and Kilbourn MR, [C-11]Glycylsarcosine: synthesis and in vivo evaluation as a PET tracer of PepT2 transporter function in kidney of PepT2 null and wild type mice. Bioorg Med Chem 2005:13;2293-3001.

Koeppe RA, Gilman S, Joshi A, Liu S, Little R, Junck L, Heumann M, Frey KA, Albin RL. Equivalence of DTBZ K1 and FDG PET measures in differentiating dementias. J Nucl Med 46:936-944, 2005.

Kuhl DE, Koeppe RA, Snyder SE. Minoshima S, Frey KA, and Kilbourn MR, In vivo butyrylcholinesterase activity is not increased in Alzheimer's synapses, Ann Neurol 2006:59;13-20

S Ahn, J A Fessler, D Blatt, A O Hero. Convergent incremental optimization transfer algorithms: application to tomography. IEEE Trans Med Imaging. 2006:25;283-96.

Shao X, Koeppe RA, Butch ER, Kilbourn MR and Snyder SE, Evaluation of 18F-labeled acetylcholinesterase substrates as PET radiotracers. Bioorg Med Chem 2005:13;869-875.

Kilbourn MR, Kemmerer E, Desmond T, Sherman P and Frey KA, Differential effects of scopolamine on in vivo binding of dopamine transporter and vesicular monoamine transporter radioligands in rat brain. Exp Neurol 2004:188;387-390. 
Kilbourn, MR. Long-term reproducibility of in vivo measures of specific binding of radioligands in rat brain. Nucl Med Biol 2004:31:591-595.

Ma B, Sherman PS, Moskwa JE, Koeppe RA and Kilbourn MR, Sensitivity of $\left[{ }^{11} \mathrm{C}\right] \mathrm{N}-$ methylpyrollidinyl benzilate $\left(\left[{ }^{11} \mathrm{C}\right] \mathrm{NMPYB}\right)$ to endogenous acetylcholine: PET Imaging vs. Tissue Sampling Methods. Nuc Med Biol 2004:31;393-397.

Albin RL, Koeppe RA, Bohnen NI, Nichols TE, Meyer P, Wernette K, Minoshima S, Kilbourn MR and Frey KA, Increased ventral striatal monoaminergic innervation in Tourette syndrome, Neurology 2003:61;1310-1315.

Albin RL, Cross D, Cornblath WT, Wald JA, Wernette K, Frey KA and Minoshima S, Diminished dtriatal [123I]iodobenzovesamicol binding in idiopathic cervical dystonia, Ann Neurol 2003:53;528-532.

Shao X, Lisi JM, Butch ER, Kilbourn MR and Snyder SE. N-Methylpiperidinemethyl, $N$-Methylpyrrolidyl and $N$-Methylpyrrolidinemethyl Esters as PET Radiotracers for Acetylcholinesterase Activity, Nucl Med Biol 2003:30;293-302.

Shao X, Butch ER, Kilbourn MR and Snyder SE, N-[18F]fluoroethylpiperidinemethyl and $\mathrm{N}-[18 \mathrm{~F}]$ fluoroethylpyrrolidinyl esters as radiotracers for acetylcholinesterase. Nuc Med Biol 2003:30;491-500

Kilbourn MR, Ma B, Butch ER, Quesada C and Sherman PS, Anesthesia increases in vivo $\mathrm{N}-\left(\left[{ }^{18} \mathrm{~F}\right]\right.$ fluoroethyl)piperidinyl benzilate $\left(\left[{ }^{18} \mathrm{~F}\right] \mathrm{FEPB}\right)$ binding to the muscarinic cholinergic receptor. Nucl Med Biol, in press 2007

\section{Published abstracts:}

Brown-Proctor C, Blair JB, Bottoms JA, Buchheimer N, Snyder SE, Butch ER, Sherman PS, Gage HD, Morton KA. Synthesis and characterization of a novel fluorine-18 labeled inhibitor of acetylcholinesterase. J Labelled Comp Radiopharm, 2003:46;S162.

Pichika R, Hu M-K, Shaw J, Snyder SE. Brain Permeation of Bis-tacrine acetylcholinesterase inhibitors measured using PET radiotracers. J Nucl Med 2003: 44;220P-221P.

Kilbourn MR and Jewett DJ, New radioligands for mu opiate receptor imaging: derivatives of [11C]carfentanil. J Cerebral Blood Flow Metab 2003:23 (Suppl 1);700.

Kilbourn MR, Sherman PS, Desmond TJ and Frey KA, Scopolamine differentially alters radioligand binding to the dopamine transporter (DAT) and vesicular monoamine transporter (VMAT2). J Cerebral Blood Flow Metab 2003:23 (Suppl 1);638.

Koeppe RA, Raffel DE, Snyder SE, Frey KA, Kilbourn MR and Kuhl DE, Interventional studies for validation of dual-[11C]tracer single-acquisition PET. J Cerebral Blood Flow Metab 2003:23 (Suppl 1);625. 
Ahn S, Fessler JA, Statistical emission image reconstruction for randoms-precorrected PET scans using negative sinogram values. Proc. IEEE Nuc. Sci. Symp. Med. Im. Conf., 2003. 